\title{
Algunas claves para la atención de estudiantes y la formación integral
}

\section{Some keys to student care and integral formation}

Leticia Rodríguez Audirac ${ }^{a}$

a Centro para el Desarrollo Humano e integral de los Universitarios, Universidad Veracruzana, Xalapa, México. Contacto: lerodriguez@uv.mx

Recibido: 29 de junio de 2020

Aceptado: 08 de octubre de 2020

RESUMEN: La formación integral pretendida por la Universidad Veracruzana, UV, ha demandado un complejo trabajo de la institución al que han ido integrándose cada vez más académicos, entidades, dependencias administrativas y de apoyo. También han sido creados programas y áreas específicas para coordinar nuevas tareas que contempla este enfoque educativo. Sin embargo, lo anterior parece no ser suficiente cuando continúan existiendo estudiantes que no reciben orientación o atención oportuna y adecuada a diversos problemas, llegando a ocasionar a un elevado número de ellos la deserción. Presentamos aquí algunas reflexiones sobre la formación integral en la UV, sobre el esquema en que se lleva a cabo, y sobre aquellas áreas de la universidad que están dedicadas a ofrecer actividades con las cuales participan en esta tarea. En ese contexto ubicamos la función del Centro para el Desarrollo Humano e Integral de los Universitarios, CEnDHIU, para sugerir las posibilidades de articulación con el sistema institucional de tutorías y con otras entidades que realizan tareas afines a las de este centro, de manera que se amplíen los servicios de apoyo de forma pertinente para estudiantes, y se contribuya a disminuir la deserción.

Palabras clave: Formación integral; trayectoria escolar; deserción.

ABSTRACT: The comprehensive training sought by the Universidad Veracruzana has demanded a complex work from the institution to which more and more academics, entities, administrative and support units have been integrated. Specific programs and areas have also 


\section{Sección especial - Centro para el Desarrollo Humano e Integral de los Universitarios}

been created to coordinate new tasks contemplated by this educational approach. However, the above does not seem to be enough when there continue to be students who do not receive timely and adequate guidance or attention to various problems, causing a high number of them to drop out. We present here some reflections on comprehensive training in the UV, on the scheme in which it is carried out, and on those areas of the university that are dedicated to offering programs and activities with which they participate in this task. In this context we place the function of the Center for Human and Integral Development of University Students, CEnDHIU, to suggest the possibilities of articulation with the institutional tutoring system and with other entities that carry out tasks related to those of this center, so that expand timely and relevant support services for students, and help reduce school dropouts.

Keywords: Integral formation: school career: school dropout.

\section{Introducción}

T a Universidad Veracruzana, ha cumplido ya veinte años de haber asumido el fin de la Lormación integral como compromiso de su tarea educativa y ha desplegado gradualmente un esquema de estrategias y acciones para lograrla. Atender integralmente al estudiante implica además de procurar el desarrollo armónico de las dimensiones profesional, intelectual, humana y social; la prevención y apoyo en los problemas que afectan la trayectoria y permanencia en la universidad. Se trata de un esfuerzo institucional enorme que ha implicado nuevas tareas para los académicos y también para los estudiantes, procesos de formación especiales, recursos extraordinarios, participación de otros actores, replanteamiento de diversas labores, nuevos espacios, etc.

Para la UV, la formación integral consiste en atender en el proceso educativo las dimensiones social y humana, además de la profesional e intelectual que ya estaban explícitas en su misión. El primer paso fue formalizar estas dimensiones en los objetivos, en los planes y programas; el segundo paso, y el más difícil, ha sido hacerlas realidad.

Se trata de una tarea compleja si reconocemos que el proceso educativo no se limita al terreno intelectual, a los conocimientos y al desarrollo de habilidades, incluye también la salud, procesos emocionales, sociales, de la personalidad, de actitudes y valores, por mencionar los 
más significativos. Además, el desenvolvimiento de estas áreas en cada persona se ve afectado por problemas familiares, económicos, por los antecedentes escolares, etc.

Al ingresar a la universidad los jóvenes que estudian licenciatura, técnico superior universitario y técnico, se ubican en su mayoría, en torno a los 18 años. Físicamente han dejado de ser adolescentes, pero se encuentran en proceso de definición de su identidad, de ejercer la toma de decisiones sobre diferentes aspectos de su vida y proyectar un plan de carrera y de vida como adultos. En esa edad son hijos, amigos, novios, estudiantes, y varios pueden ser ya trabajadores o padres. Muchos de ellos están por primera vez viviendo fuera de su casa, en otros la transición hacia la autonomía y la responsabilidad de su vida les presenta dificultades que se suman con su llegada e integración a la vida universitaria.

Adrián De Garay, uno de los investigadores dedicado a la investigación de los estudiantes, aborda el fenómeno de la integración a la vida universitaria en su artículo La integración académica y cultural a la universidad de los jóvenes universitarios, publicado en el año 2016 en la Revista CLABES de la UNAM. De Garay advierte la importancia de la etapa de adaptación a la universidad por el hecho de que la mayor deserción en educación superior ocurre en el primer año de escuela, tal como lo reportan los indicadores de prácticamente todas las universidades.

De Garay hace notar que los jóvenes enfrentan las exigencias de ser universitarios en el reconocimiento social, intelectual y en las capacidades y hábitos de trabajo a lo largo de sus estudios de licenciatura.

En las conclusiones de esta investigación, Adrián de Garay señala que el capital cultural con el que la persona llega a la universidad si tiene un peso importante en el proceso de integración, sin embargo, dice

“...En la medida que la institución universitaria se conciba como un espacio donde los jóvenes no sólo acuden a sus aulas a nutrirse de conocimientos, sino también como un espacio privilegiado donde pueden tener contacto y formarse en torno a distintas expresiones culturales, las posibilidades de integración académica son mayores." (De Garay 2016) 


\section{Sección especial - Centro para el Desarrollo Humano e Integral de los Universitarios}

En síntesis, De Garay revela cuatro factores como los que mayor influencia tienen en el éxito de la integración a la vida universitaria. Dos propios del estudiante, es decir independiente de la escuela, se trata del capital cultural con el que llega el estudiante y de los habitus culturales, que, para el caso del estudio referido, se encontró que un menor consumo de televisión favorece la integración. Los otros dos factores ocurren al interior de la universidad, uno es el consumo que el joven hace de la oferta cultural de la institución, sobre todo en el primer año, y el otro está en las prácticas pedagógicas de un determinado grupo de docentes. De Garay termina destacando la importancia de cuidar especialmente en el primer año, la integración social y académica de los estudiantes para reducir el rezago y la deserción escolar. De acuerdo con sus hallazgos, lo que habrá de fortalecerse es que los alumnos participen y consuman la oferta de experiencias culturales y sociales que la universidad genera.

Retomamos también de la investigación realizada por Alarcón (2017) sobre el consumo cultural de estudiantes de ocho programas de la UV, la idea de que es necesario partir de que los jóvenes son un grupo heterogéneo, por lo que no es posible tener ni los estereotipos anteriores de lo que era ser universitario, ni un solo perfil para definirlos. En sus conclusiones señala que “...es posible identificar pautas de consumo que no se explican completamente a partir del origen social (...) no obstante -y pese a que se observa cierto eclecticismo en sus prácticas de consumo (...) las diferencias socioeconómicas y culturales siguen estando presentes." (Alarcón, 2017).

Por lo tanto, además de reconocer la diversidad de los perfiles de los alumnos y no perder de vista el peso de su condición socioeconómica cultural, el seguimiento y cuidado de los estudiantes al interior de la universidad es un factor fundamental y puede ser determinante cuando se quiere evitar la deserción y procurar su formación integral. Cuidar especialmente el primer año de los jóvenes en la universidad sin duda es una clave importante para disminuir la deserción, pero lo es también para emprender con mayor éxito, el proceso de acompañamiento, orientación y apoyo a las cuatro dimensiones que han de ser impulsadas. Otra clave, está en que la universidad ofrezca a los estudiantes las suficientes opciones de actividades y experiencias del ámbito cultural y de participación interpersonal. Veamos ahora de qué manera la UV contempla claves como las aquí señaladas. 


\section{¿Cómo se atiende la formación integral en la UV?}

Trabajar por este fin ha significado el despliegue de numerosas tareas en la institución que inician con incluir en los planes de estudio, la formalización de cursos y actividades de artes, deportes, salud, así como disciplinas diferentes a las del programa educativo. Además, ha sido importante la construcción y desarrollo de un sistema de tutorías para acompañar y orientar la trayectoria de cada estudiante, la innovación de la docencia, la participación de los académicos como tutores, etc. Sin embargo, aun cuando ha implicado el trabajo de los actores y funciones prácticamente de todos los ámbitos de la institución y, hay cambios importantes; las evaluaciones que se han realizado al modelo educativo de la UV, muestran que el interés, la atención y la evaluación en los programas educativos, está centrada en los saberes disciplinares, teóricos y metodológicos correspondientes a la profesión, dejando de lado el eje axiológico, y las actividades para el desarrollo de las dimensiones social y humana.

La formación integral está declarada en los objetivos generales y específicos de cada programa educativo, está definida en los planes de estudio y se propone sea desarrollada de forma articulada en cada experiencia educativa o asignatura.

También se determinó una de las cuatro áreas que tienen todos los planes de estudio, el Área de Formación de Elección Libre, como el espacio en el que el estudiante puede dedicarse al aprendizaje de saberes de disciplinas distintas a las de la carrera que estudia. Para el funcionamiento de esta área la Universidad ha creado una amplia oferta de cursos diversos con valor crediticio en artes, salud, desarrollo humano, ética y valores, ciencia, deportes, idiomas, sustentabilidad, género, etc.

Una estrategia fundamental para la formación integral es la tutoría que, en la UV, se ha desarrollado en un esquema de modalidades diversas. La tutoría abarca cuatro dimensiones, académica, profesional, personal y de integración y pertenencia. La académica consiste en el acompañamiento y orientación a lo largo de su trayectoria escolar, la profesional en la orientación en las decisiones de la conformación de su perfil profesional y ético. La personal contribuye al desarrollo de valores, la toma de decisiones de forma autónoma, responsable e 
informada, y la de integración y pertenencia, fomenta el sentido de responsabilidad y el compromiso con su educación.

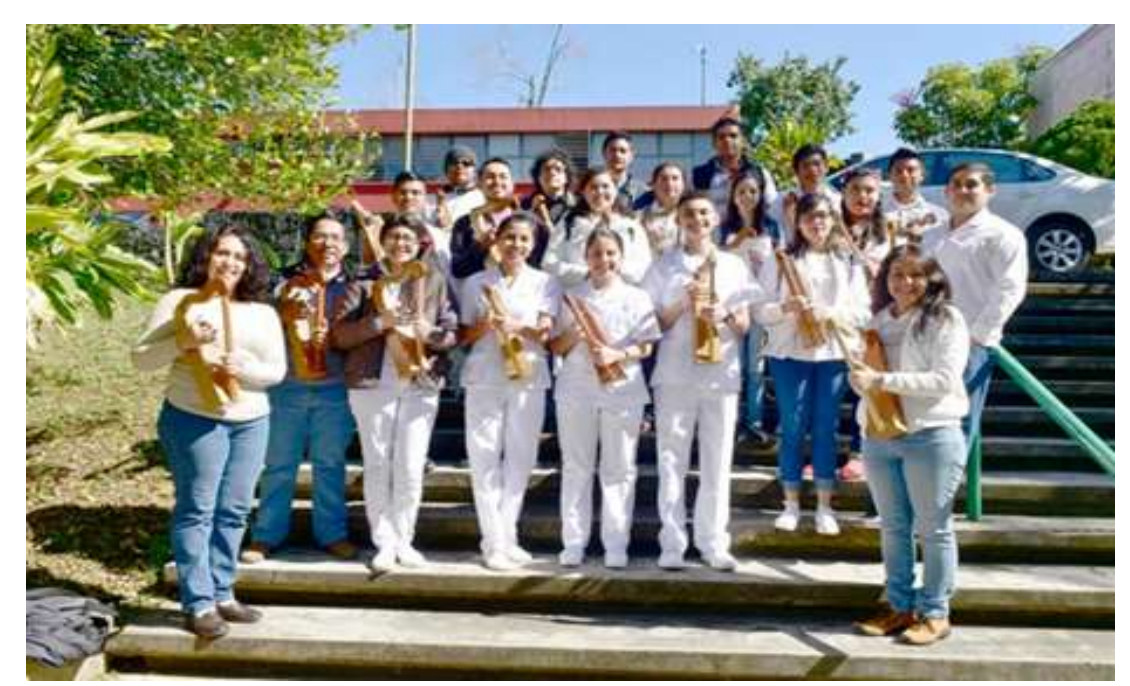

Figura 1. Alumnos de la UV. Fuente: Periódico Universo.

https://www.uv.mx/prensa/general/tutorias-para-la-apreciacion-

artistica-potencian-el-talento-universitario

Gradualmente se fueron desarrollando otras modalidades de tutoría que buscan fortalecer un mayor número de dimensiones, así la enseñanza tutorial atiende de manera personalizada a los estudiantes que enfrentan dificultades específicas con las experiencias educativas o asignaturas que cursan. La tutoría para la apreciación artística la define su nombre, pretende desarrollar un sentido crítico y de valoración para diferentes expresiones del arte. La tutoría para la investigación promueve el desarrollo del interés por la ciencia y por la investigación incorporando a los estudiantes en proyectos de investigación. Una modalidad más es el programa de monitores mediante el cual, estudiantes de semestres superiores apoyan a sus pares de primeros semestres en sus asignaturas, procurando además promover el trabajo en equipo, habilidades de comunicación y liderazgo. Asociado todo lo anterior, en la UV se lleva a cabo un evento anual para recibir a los estudiantes de nuevo ingreso y apoyar su integración a la vida en la institución a través de la inducción, información y orientación sobre lo que significa ser universitario, mostrándole la oferta de experiencias educativas, servicios diversos que puede cursar y los de apoyo, la propuesta cultural, deportiva, la normatividad, etc. "Conoce tu Universidad" es el nombre con el que se denominan estas actividades. 
Sin duda el funcionamiento de este esquema de tutoría requiere de un proceso permanente de monitoreo y de evaluación para su transformación constante y mejora, pero sobre todo para asegurar que los estudiantes estén recibiendo la orientación y ayuda, para evitar la deserción y para contribuir en la formación de manera integral. Con el desarrollo de este sistema se ha logrado alcanzar la atención de es 48,024 Tutorados (de acuerdo con el informe de labores de la UV 2019), contando con la participación de 2,928 Tutores. Es decir, en la UV el 78\% Cobertura de alumnos de licenciatura, técnico superior universitario y técnico, reciben tutoría.

Las universidades como la UV tienen grandes ventajas para procurar el desarrollo de todas las dimensiones ya que además de un esquema de tutorías como el que se ha logrado desarrollar, con la participación de los académicos, existen las facultades e institutos especializados en disciplinas diversas que enriquecen la formación integral. Así mismo la existencia de prestigiados grupos artísticos, ha hecho posible la participación de artistas impartiendo experiencias educativas y orientación en tutoría, y ni se diga de la permanente oferta cultural que la universidad tiene. En el área de deportes y cuidado de la salud, la UV ya contaba con dependencias que ofrecían actividades y asesoría al respecto, para participar formalmente en el currículo, asignó valor en créditos a tales actividades y amplió la oferta significativamente. Un enfoque educativo como el de la UV también contempla el aprendizaje de idiomas con valor curricular para lo que existe actualmente un sistema de centros de idiomas y auto acceso con modalidades flexibles y autónomas, con una oferta amplia para el aprendizaje de lenguas que incluyen los idiomas originarios de México. Los programas de internacionalización, así como los diez tipos diferentes de becas económicas a las que los estudiantes tienen acceso (cinco institucionales y cinco externas), contribuyen con los mismos fines de formación.

Por otro lado, de manera complementaria, en la UV existen instancias dedicadas a la promoción de la salud, asesoría psicológica y psicopedagógica. Una de ellas es el Centro para el Desarrollo Integral y Humano de los Universitarios, CEnDHIU. Está ubicado en la región Xalapa, tiene como tarea impulsar la cultura de estilos de vida saludables en la comunidad universitaria a través de guías para el autocuidado, la orientación psicológica y nutricional, para informar y orientar sobre cómo adquirir hábitos saludables y sobre la responsabilidad 


\section{Sección especial - Centro para el Desarrollo Humano e Integral de los Universitarios}

de la sexualidad sin riesgos, para promover el desarrollo humano, brindar apoyos pedagógicos y atender a estudiantes con discapacidad.

Si bien existen ahora más alternativas para el desarrollo de las dimensiones humana y social, no son suficientes porque los obstáculos en la vida universitaria que ponen en riesgo la trayectoria estudiantil ocasionando la deserción, corresponden a factores de las cuatro dimensiones de la formación integral, que generan una interacción compleja. De éstas, la social y la humana, requieren de estrategias que no están previstos con la misma suficiencia como lo están aquellos que generalmente vemos asociados con el desarrollo profesional y un poco, con el intelectual. Se trata de un grave problema de la educación que también es complejo y que hay que observarlo en el contexto de la educación de un país.

Al estar centrados en las tareas académicas y en los resultados de la formación profesional, ignorando nuestra responsabilidad con la formación integral, se corre el riesgo de que se asuma como una situación normal, el que la matrícula de cada generación en la universidad vaya disminuyendo conforme se avanza en los semestres. En una situación así, se desestima la atención y apoyo a los estudiantes, que, para cada caso, la situación es distinta, pudiendo ser de origen externo e interno a las universidades, previos a los estudios superiores en muchos casos, pero de responsabilidad de todos los que forman parte de una institución que tiene como fin educativo este tipo de formación que da relevancia también a lo humano y lo social.

Para dimensionar la gravedad de la deserción escolar, hay que recordar primero el esfuerzo por ingresar a la educación superior. Durante los meses de mayo y junio de cada año reaparece en los medios el tema de ingreso a la Universidad Veracruzana, durante ese tiempo las cifras de los jóvenes que aspiran a ingresar y las de aquellos que logran alcanzar uno de los 17 mil lugares aproximados que se ofrecen, son noticia casi cotidiana que contribuye a mantener presente algunos de los rasgos de la educación en México que no se han podido resolver, la cobertura y el acceso a la educación superior.

Si bien las universidades centran sus tareas en alcanzar sus fines y objetivos declarados en torno a la docencia, la investigación y la vinculación; no pueden hacerlo sin dejar de dedicar acciones concretas para evitar que esos jóvenes que ya lograron un lugar en la universidad sean expulsados por las múltiples causas que originan la deserción. 
Y es que de esos grandes problemas que tiene México en materia educativa, la cobertura y acceso, se van agravando conforme de mayor nivel educativo se trata. El paso de las generaciones de niños y jóvenes por cada nivel educativo del sistema va dejando fuera a un gran número de ellos para quienes la posibilidad regresar a estudiar prácticamente desaparece.

Para ilustrar de forma rápida lo dicho anteriormente, retomamos algunos datos de la educación en México, de años recientes. La escolaridad en México, en el 2015, se reportó en 9.2 años, de acuerdo con el promedio de grados de escolaridad de la población de 15 años y más. En tanto que la población adulta de entre 25 y 64 años con estudios de educación superior, en el mismo año referido, correspondía a 16 de cada 100 adultos del rango. En este último indicador y en porcentaje de adultos con maestría, que es de 1\% en nuestro país, México está en el último lugar de los países de la OCDE.

Por su parte de acuerdo con datos de la UNESCO del año 2018, la cobertura de educación superior en México, es decir, el porcentaje de personas entre 19 y 23 años cursando estudios superiores, era del 38.4\%, mientras que países de América Latina ya alcanzaban porcentajes de 58.7\% en Colombia por ejemplo o, en Argentina 85.7\% y 90.3\% en Chile. De acuerdo con lo reportado por la ANUIES, Veracruz presentaba en los años 2017-2018, 32.6\% de cobertura de educación superior.

Esta brecha en cobertura junto con la brecha que significa el abandono de estudios de nivel superior plantea un problema aún más grave. De acuerdo con los datos de la Gaceta Oficial del Estado de Veracruz, 2019, en Veracruz 14.5\% de los que ingresan a este nivel educativo, abandona la escuela. Sin embargo, los datos del Instituto Nacional de Estadística y Geografía (INEGI), indican que solamente 8 de cada 100 alumnos que ingresa a la universidad logra concluir, dato que es muy bajo, hemos de suponer que este último, solo se refiere a sistemas universitarios, porque así lo denominan en INEGI, dejando fuera tecnológicos, institutos, etc. También de acuerdo con la información del INEGI, los principales motivos de deserción en nivel universitario apuntan al poco interés en el estudio o estado de disgusto (37.4\%), y por situaciones económicas (35.2\%). 
Para la Universidad Veracruzana el primer desafío es la alta demanda de ingreso que año con año tiene para licenciatura y técnico superior universitario, en el año 2019 rebasó los 45 mil aspirantes. La capacidad de esta institución le permite admitir al 35\% de dicha demanda. Actualmente la UV atiende a uno de cada cuatro estudiantes de nivel superior en Veracruz, así participa en la tarea por atención de la demanda de educación superior.

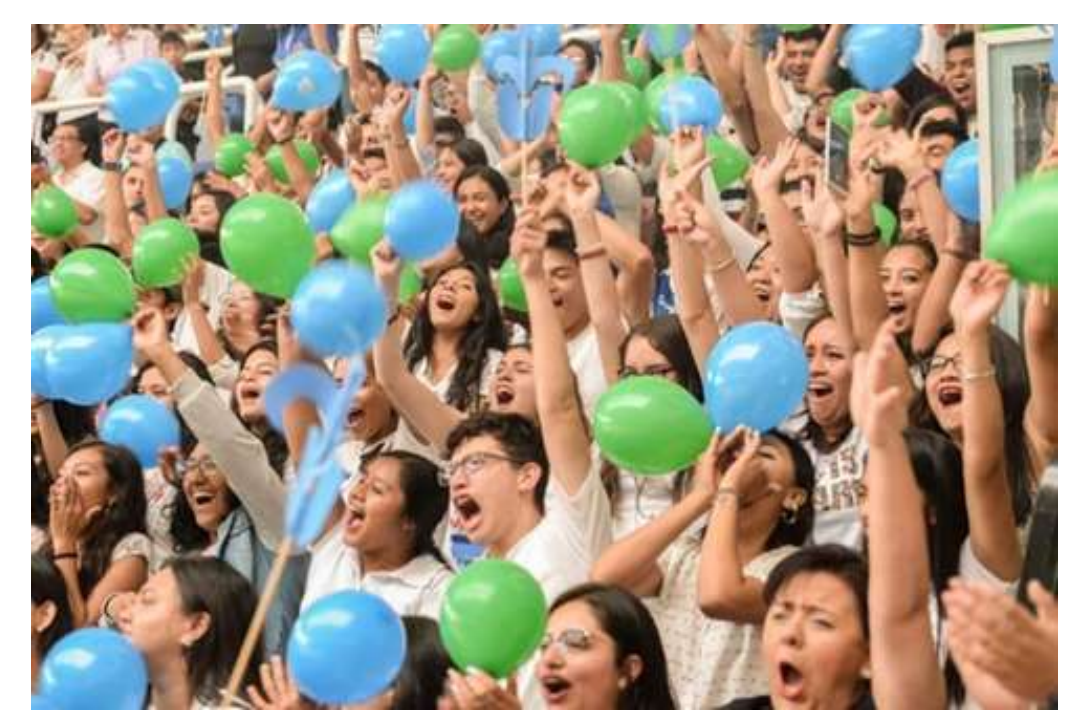

Figura 2. Alumnos de la UV. Fuente: Periódico Universo.

https://www.uv.mx/prensa/banner/ingreso-a-la-uv-la-generaciondel-75-aniversario/

Con los datos anteriores podemos conocer un poco sobre la dimensión del compromiso de una institución como la UV, que además de alcanzar sus fines educativos, tiene la responsabilidad de evitar que los pocos jóvenes que ya han logrado llegar a este nivel educativo, abandonen sus estudios. Y es que prácticamente todas las instituciones de educación superior públicas tienen cifras muy bajas de graduados en relación con el número de estudiantes que ingresan. Veamos el caso de la UV en la que el número de egresados en el año 2019 fue 7,215. Para calcular el porcentaje de egresados que tal cifra representa, tomamos como referente la matrícula de nuevo ingreso del año 2014, es decir cinco años atrás como el tiempo promedio en el que se cursa un programa educativo. En ese año se inscribieron 15,429 jóvenes de recién ingreso, por lo que calculamos el porcentaje de egreso en $46.76 \%$ y el de deserción en 53.24\%. Sería ideal que las universidades públicas alcanzaran sus fines de educación, los objetivos de cada carrera, y el egreso de todos los jóvenes que llegan a estudiar 
cada año. Actualmente en la UV el proceso de ingreso ofrece más de 17 mil lugares para ingresar, es decir no cesan los esfuerzos por aumentar la cobertura, pero múltiples factores intrínsecos y extrínsecos continúan impidiendo una trayectoria adecuada a muchos estudiantes y provocando el abandono de un alto número de jóvenes.

Interesa recordar de qué idea partimos, La SEP define a la deserción escolar como el abandono de las actividades correspondientes antes de terminar algún grado o nivel educativo (SEP 2004), tiene múltiples causas, y ha sido motivo de estudios diversos, la mayoría de ellos, para conocer las causas y orientar a las instituciones educativas en el diseño de acciones y apoyos para prevenir y evitarla.

La mayor deserción en el nivel superior ocurre en el primer y segundo año de estudios. De acuerdo con la investigación realizado por Dzay y Narváez, el abandono ocurre en la mayoría de las veces tras un alto índice de reprobación en los dos primeros años, pero lo que está detrás de ello pueden ser situaciones diversas, que no están necesariamente asociadas a la dificultad de las asignaturas.

...algunas razones que podrían impactar en la deserción escolar, a saber: lugar de origen lejano a la universidad, problemas familiares, falta de recursos económicos, espacios inadecuados para estudiar, necesidad de laborar mientras estudian, problemas de salud o adicciones, falta de competencias básicas previas a su ingreso a la universidad, alto grado de exigencia por parte de los profesores y la elección inadecuada de la carrera. (Dzay y Narváez, 2012: 20)

Las causas por las que dejan sus estudios señaladas por los autores de esta investigación coinciden con los resultados de numerosos trabajos en los que se ha estudiado el problema (Caballero, Reyes, Rodríguez y Bolívar, 2016; Rodríguez, Posada, Velásquez y Estrada, 2016), en ellos se ha concluido que en la deserción los factores presentes son: una edad mayor a 26 años, menor nivel de motivación intrínseca, insatisfacción con la carrera elegida, menor satisfacción con la funcionalidad de su familia y bajo uso de estrategias de aprendizaje y deficientes hábitos de estudio.

Situaciones como baja autoestima, consumo de drogas, violencia familiar o de pareja, depresión, embarazos no planeados, elección de carrera errónea, necesidad de trabajar, 


\section{Sección especial - Centro para el Desarrollo Humano e Integral de los Universitarios}

deficiencias en los conocimientos adquiridos, entre otros, son los principales problemas por los que los estudiantes están en riesgo de abandonar sus estudios. Éstos no pueden ser ignorados en una institución que además de pretender el egreso, le interesa el desarrollo y la formación armónica de los estudiantes en todas sus dimensiones como persona: profesional, intelectual, humana y social.

Por las razones anteriores, y por la creciente demanda por parte de los alumnos, de apoyos de orientación para el cuidado de su salud, para su desarrollo emocional, humano y social; se ha hecho evidente la importancia que tienen los servicios que en estos ámbitos ofrecen las instituciones para contribuir a una mejor integración a la universidad, a evitar la deserción y a lograr la formación integral.

Los estudiantes de la UV como todos los en el país tienen derecho a los servicios de salud que, en el caso de los universitarios, son los del Instituto Mexicano del Seguro Social. Como sabemos el esquema de estos servicios consiste en tratar o curar enfermedades. Sin embargo, el enfoque de salud en la formación integral está orientado a la promoción de estilos de vida saludables en los que se parte de informar y orientar para fortalecer la autonomía y responsabilidad en el cuidado físico, mental y emocional, como una condición necesaria para del desarrollo armónico de todas las dimensiones de la persona.

Así, mientras se avanza en la institución en articular el andamiaje educativo en el currículo para el desarrollo armónico de las dimensiones humana y social junto con la intelectual y profesional, todos los apoyos que se genere para prevenir o atender problemas, estarán contribuyendo con el fin educativo planteado.

\section{¿Qué apoyos tiene la UV para promover la salud?}

La capacidad de la institución para promover la salud presenta brechas que no han podido ser resueltas, de manera que es en la Región Xalapa en la que existe el Centro para el Desarrollo Humano e Integral de los Universitarios, CEnDHIU. En éste se llevan a cabo programas y se brindan servicio con una alta demanda por parte de todos los integrantes de la comunidad universitaria, siendo los estudiantes, los más asiduos. Cuenta con el espacio de la propia entidad y diez Centros Centinela que han sido instalados estratégicamente en las 
facultades a fin de constituirse en un espacio de contacto para que los estudiantes accedan y participen en las actividades.

La Región Xalapa tiene una matrícula de 25,206 estudiantes de licenciatura, técnico superior universitario y técnico. El CEnDHIU atiende en promedio 5,000 estudiantes al año que acuden por alguno de los servicios que se ofrecen, asesoría psicológica, orientación nutricional, participación en talleres sobre salud, autoestima, manejo del estrés, conflictos o violencia en el noviazgo, riesgos del consumo de drogas, salud y sexualidad, pruebas de VIH y adquisición de métodos anticonceptivos. También asisten a las ferias de salud. Es decir, mientras para algunos el apoyo puede ser de una sola ocasión, otros son usuarios de más de un servicio. Si consideráramos que los 5 mil estudiantes atendidos anualmente corresponden a 5 mil personas diferentes, podríamos calcular una cobertura de atención en promoción de la salud del 20\%. Es decir, en Xalapa, son usuarios del CEnDHIU, cuando mucho 20 de cada 100 estudiantes. En el caso de las otras Regiones de la universidad, es Veracruz, la que tiene una entidad que brinda apoyos en salud, las demás, no cuentan con un espacio como el CEnDHIU.

Hemos dicho líneas antes, que una estrategia muy importante para apoyar la formación integral es el acompañamiento en su trayectoria escolar. Todos los estudiantes de la UV tienen derecho a recibir la tutoría, para lo que les es asignado un tutor académico que realiza dicha tarea. Cada facultad tiene organizado un sistema que agrupa a los tutores de la entidad y trabaja en la planeación, seguimiento y evaluación de esta actividad. Con esta estrategia se da seguimiento a los alumnos para advertir los riesgos y obstáculos que tienen, para dirigirlos a las instancias correspondientes de acuerdo con cada situación, quizá para conseguir una beca económica, para ser atendido por los especialistas psicólogos o médicos, para realizar un estudio vocacional, tomar cursos específicos, etc. Derivado de esta actividad, en el último informe reportado por el Sistema Institucional de tutorías de la UV, los tutores señalan que las dificultades de mayor recurrencia manifestados por los estudiantes son situaciones de discriminación, de bullying, debilidad en competencias interpersonales, en motivación, carencia de hábitos de estudio y de capacidad de toma de decisiones.

En la búsqueda de una mejor articulación de las actividades con las que en la UV se atiende a los alumnos en diversas problemáticas, la coordinación del Sistema Institucional de Tutorías 


\section{Sección especial - Centro para el Desarrollo Humano e Integral de los Universitarios}

y el CEnDHIU, están trabajando por establecer mecanismos de comunicación y seguimiento que faciliten una mayor participación en los programas y servicios del CEnDHIU.

Por otro lado, en la búsqueda de unir más esfuerzos para un mejor desenvolvimiento en la universidad, el CEnDHIU, y el Instituto de Psicología y Educación que entre sus funciones lleva a cabo acciones de atención psicoeducativa, y que es poseedor de una valiosa experiencia; están dialogando para definir también mecanismos que posibiliten coordinarse entre ellos y con el Sistema Institucional de tutorías, para extender las opciones de apoyo para los estudiantes.

Estamos seguros de que, en la Región Xalapa, aún hay más entidades y académicos cuyo quehacer y área de especialidad, contribuiría en atender las necesidades que presentan los jóvenes durante sus estudios en la universidad, particularmente en las dimensiones humana y social. En las otras cuatro regiones, que tienen una conformación diferente y un menor número de entidades y dependencias de apoyo, es aún más necesario fortalecer un trabajo articulado y en red para la formación integral y ofrecer alternativas para un mejor desarrollo de los estudiantes. Prácticamente todas las facultades y los institutos dedicados a las ciencias de la salud realizan acciones de extensión en odontología, medicina, enfermería y psicología, en tanto que las facultades de pedagogía ofrecen asesorías psicopedagógicas. Ante la necesidad de ampliar y fortalecer todos los esfuerzos por abatir la deserción y alcanzar los objetivos educativos planteados, esos que son servicios de extensión hasta ahora, podrían organizarse para trabajar en red de apoyo con los tutores de todas las facultades de la región y orientarse con prioridad a los alumnos de la UV, y a los problemas emergentes que los están afectando.

\section{Referencias}

Alarcón G. José. (2017) ¿DISTINCIÓN U OMNIVORIDAD? CONSUMO CULTURAL DE LOS ESTUDIANTES DE NUEVO INGRESO EN LA UNIVERSIDAD VERACRUZANA Clivajes. Revista de Ciencias Sociales. Año IV, número 8, julio-diciembre, 2017, pp. 166-193. Instituto de Investigaciones Histórico-Sociales Universidad Veracruzana. México Disponible en http://clivajes.uv.mx/index.php/Clivajes/article/view/2535/4417 
Sección especial - Centro para el Desarrollo Humano e Integral de los Universitarios

ANUIES. Visión y acción 2030. Propuesta de la ANUIES para renovar la educación superior en México Diseño y concertación de políticas públicas para impulsar el cambio institucional. Asociación Nacional de Universidades e Instituciones de Educación Superior. 2018. México. Recuperado en http://www.anuies.mx/media/docs/avisos/pdf/VISION_Y_ACCION_2030.pdf

Caballero Domínguez, C., Reyes Sarmiento, M., Rodríguez Pautt, A., \& Bolivar Troncoso, A. (2016). Factores de Riesgo Sociodemográficos, Psicosociales y Académicos de Abandono de los estudios en Estudiantes de Primer Semestre de la Universidad del Magdalena, Colombia*. Congresos CLABES. Recuperado de https://revistas.utp.ac.pa/index.php/clabes/article/view/869

Rodríguez, M., Posada, M., Velásquez, M., \& Estrada, P. (2016). Población con riesgo de abandono universitario. Una aproximación desde la prevención. Universidad de Antioquía. Congresos CLABES. Recuperado de https://revistas.utp.ac.pa/index.php/clabes/article/view/873

De Garay, A. (2016). La integración académica y cultural a la universidad de los jóvenes universitarios. Un modelo de análisis y la implementación de políticas institucionales. Congresos CLABES. https://revistas.utp.ac.pa/index.php/clabes/article/view/925

Dzay Ch., F. y Narváez T, O. (2012). La Deserción Escolar desde la perspectiva estudiantil. Yucatán: La Editorial Manda. https://www.uv.mx/personal/onarvaez/files/2013/02/Ladesercion-escolar.pdf

Gaceta Oficial. Órgano del Gobierno del Estado de Veracruz de Ignacio de la Llave. Tomo CC. Núm. Ext. 356. PROGRAMA SECTORIAL VERACRUZANO DE EDUCACIÓN 2019-2024. Jueves 5 de septiembre de 2019. http://www.editoraveracruz.gob.mx/gacetas/2019/09/Gac2019356\%20Jueves\%2005\%20TOMO\%20VII\%20Ext.pdf

Instituto Nacional de Estadística Geografía e Informática. Recuperado en

https://www.inegi.org.mx/app/tabulados/pxwebv2/pxweb/es/Educacion/Educacion/E ducacion_11.px/table/tableViewLayout2/

Organización de las Naciones Unidas para la Educación la Ciencia y la Cultura. (2018). Bases de datos de UNESCO. Institute for Statistics. Recuperado en http://data.uis.unesco.org Universidad Veracruzana. Series históricas 2019-2020. https://www.uv.mx/informacionestadistica/files/2019/01/Series-Historicas-2019_2020.pdf 
Universidad Veracruzana Anuario 2019. Resumen estadístico.

https://www.uv.mx/informacion-estadistica/files/2019/01/03-

Resumen_Estadistico_2019.pdf

Universidad Veracruzana. I Informe de actividades 2013 - 2014. Tradición e innovación. https://www.uv.mx/documentos/files/2019/05/1er-informeUV-2013-2014.pdf

Universidad Veracruzana. La UV en números. https://www.uv.mx/informacionestadistica/files/2019/06/UV-en-numeros.pdf

Universidad Veracruzana. Indicadores de gestión. https://www.uv.mx/informacionestadistica/files/2020/01/Indics_Gestion_MAR-2020.pdf

Universidad Veracruzana. Informe de Labores UV 2019. https://www.uv.mx/documentos/files/2019/09/20-Informe-de-Actividades-20182019.pdf

Caballero Dominguez, C., Reyes Sarmiento, M., Rodríguez Pautt, A., \& Bolivar Troncoso, A. (2016). Factores de Riesgo Sociodemográficos, Psicosociales y Académicos de Abandono de los estudios en Estudiantes de Primer Semestre de la Universidad del Magdalena, Colombia*. Congresos CLABES. Recuperado de https://revistas.utp.ac.pa/index.php/clabes/article/view/869 